Ressourcenökonomie in der Tierwirtschaft

\title{
Wirkungsgrad und Bedarf an tierischer Nahrung
}

Ein Weg, die Ernährungssicherheit weltweit zu erhöhen, besteht darin, die verfügbare landwirtschaftliche Fläche effizient zu nutzen. Unterschiedliche Nahrungsmittel unterscheiden sich dabei hinsichtlich ihres Ressourcenverbrauchs. Wie effizient ist eine Flächennutzung, um tierische Nahrungsmittel zu produzieren?

Von Tobias Gaugler

$\mathrm{n}$ Fortsetzung der Millenniumsziele konkretisieren die Vereinten Nationen aktuell ihre Nachhaltigen Entwicklungsziele, deren Priorisierung auf Basis einer Umfrage unter den Mitgliedsstaaten durchgeführt wurde. Hierbei wurde „Ernährungssicherheit und nachhaltige Landwirtschaft" als das global wichtigste Ziel identifiziert (UN 2012, S.8). Vor dem Hintergrund, dass 808 Millionen nicht genug zu essen haben, täglich mehrere zehntausend Menschen an Hunger sterben (WEP 2014) und die Weltbevölkerung weiter anwachsen wird, ist dieses Ergebnis wenig überraschend. Auch Deutschland trägt direkt zum Hunger in ärmeren Weltregionen bei, da der Eigenbedarf an Nahrungsmitteln erst durch einen Netto-Import von 18,8\%, bezogen auf die heimische landwirtschaftliche Nutzfläche, gedeckt werden kann (Meier et al. 2014, S. 28).

\section{Wirkungsgrad tierischer Lebensmittel}

Ein Großteil der landwirtschaftlichen Nutzfläche wird zur Fütterung und
Haltung von Tieren beansprucht. In Deutschland sind dies 69\% (Meier et al. 2014, S. 26), weltweit sogar $78 \%$ (LEAD 2006, S.74). Zur Bestimmung des gesamten Ressourcenverbrauchs sind neben dem Flächenverbrauch auch Faktoren wie der Wasserverbrauch, die Emission an $\mathrm{CO}_{2}$-Äquivalenten und der Einsatz von Düngemitteln zu untersuchen (Eshel et al. 2014), worauf jedoch hier aus Platzgründen verzichtet wird. Im Resultat werden große Teile der Ernte, beispielsweise $61,1 \%$ der EU-27-Getreideernte (FNR 2014, Ernte 2011/12), zur Fütterung von Tieren genutzt. Dem direkten menschlichen Konsum stehen diese pflanzlichen Nahrungsmittel somit nicht mehr zur Verfügung.

Im Folgenden soll auf die in Kalorien gemessene Energiebilanz eingegangen werden, die sich aus der Produktion von Lebensmitteln tierischen Ursprungs ergibt. Je nach Nahrungsmittel bedarf es eines unterschiedlich hohen Energieeinsatzes, um eine für den Menschen nutzbare Kalorie zu erhalten. Eshel et al. (2014) berechnen, dass dieses Verhältnis bei der Produktion von Rindfleisch durchschnittlich 36 zu eins beträgt; zur Herstellung einer Kalorie Schweinefleisch müssen elf Kalorien aufgewendet werden; um eine Kalorie Hühnerfleisch zu erhalten, sind neun Kalorien nötig. Für die Gewinnung von für den Menschen nutzbarer Energie aus Eiern und Milch ist jeweils die circa sechsfache Menge an Energie nötig (Eshel et al. 2014, S. 11999). Wenngleich sich diese Zahlen auf den US-amerikanischen Raum beziehen und andere Untersuchungen zu graduell unterschiedlichen Resultaten kommen, lässt sich vereinfachend festhalten, dass der durchschnittliche energetische Wirkungsgrad der Fleischproduktion bei etwa $10 \%$ und der der Produktion von Eiern und Milch zwischen $15 \%$ und $20 \%$ liegt.

Die bisherigen Darstellungen zusammenfassend wird der weit überwiegende Teil der landwirtschaftlichen Nutzfläche für die Viehzucht und -haltung genutzt. Von den auf dieser Fläche angebauten pflanzlichen Nahrungsmitteln wiederum geht der weit überwiegende Teil energetisch verloren, da er von einem Tier verstoffwechselt wird. Folglich steht lediglich ein sehr geringer Teil der ursprünglichen Energie dem Menschen in Form von Fleischwaren, Milchprodukten oder Eiern zur Verfügung, was aus ressourcenökonomischer Perspektive als ineffizient bezeichnet werden kann.

\section{Konsum tierischer Lebensmittel und tatsäch- licher Bedarf}

Im Folgenden soll der Verbrauch von Nahrungsmitteln tierischen Ursprungs mit dem Bedarf verglichen werden. Während die Erhebung von Konsumdaten

\begin{tabular}{|c|c|c|c|c|}
\hline & Pro-Kopf-Konsum (2011) & Empfehlung der DGE & $\begin{array}{l}\text { Mittelwert der } \\
\text { DGE-Empfehlung }\end{array}$ & Über-/Unterkonsum \\
\hline Getreideerzeugnisse & $96,5 \mathrm{~kg}$ & $73,0 \mathrm{~kg}$ bis $113,2 \mathrm{~kg}$ & $93,1 \mathrm{~kg}$ & $+4 \%$ \\
\hline Reis, Kartoffeln u. Ä. & $71,9 \mathrm{~kg}$ & $54,8 \mathrm{~kg}$ bis $91,3 \mathrm{~kg}$ & $73,0 \mathrm{~kg}$ & $-2 \%$ \\
\hline Gemüse und Obst & $206,5 \mathrm{~kg}$ & $237,3 \mathrm{~kg}$ & $237,3 \mathrm{~kg}$ & $-13 \%$ \\
\hline Fleisch(-erzeugnisse) & $35,4 \mathrm{~kg}$ (Frauen) bis 58,0 kg (Männer) & 15,6 kg (Frauen) bis 31,2 kg (Männer) & $23,4 \mathrm{~kg}$ & $+100 \%$ \\
\hline Milch(-erzeugnisse) & $154,0 \mathrm{~kg}$ & $91,3 \mathrm{~kg}$ bis $113,2 \mathrm{~kg}$ & $102,2 \mathrm{~kg}$ & $+51 \%$ \\
\hline Eier & 217 Stück & max. 156 Stück & $\max .156$ & mind. $+39 \%$ \\
\hline
\end{tabular}

Tabelle 1: Abweichung des jährlichen Pro-Kopf-Verbrauchs an Nahrungsmitteln (BMEL 2013, S. 189-193)

von den Empfehlungen der Deutschen Gesellschaft für Ernährung (DGE 2014) 
leicht möglich ist (BMEL 2013, S. 189193), divergieren Aussagen über den aus gesundheitlicher Perspektive sinnvollen Bedarf. Etablierte Organisationen wie die Deutsche Gesellschaft für Ernährung (DGE) erachten eine Ernährung als sinnvoll, die auch tierische Produkte umfasst (DGE 2014). Demgegenüber legen aktuelle Untersuchungen nahe, dass neben fleischfreier auch eine vegane Ernährung gesundheitlich positive Auswirkungen hat (Esselstyn et al. 2014).

Um Aussagen zur Über- beziehungsweise Unterversorgung mit verschiedenen Gruppen von Lebensmitteln tätigen zu können, soll nun der durchschnittliche Pro-Kopf-Verbrauch mit dem tatsächlichen Bedarf verglichen werden (siehe Tabelle 1). Als Obergrenze für den Konsum von Nahrungsmitteln tierischen Ursprungs werden hierbei die Mittelwerte der Empfehlungen der DGE gewählt. Es wird deutlich, dass der durchschnittliche Konsum von Nahrungsmitteln tierischen Ursprungs in Deutschland weit über allen Empfehlungen liegt. So sollte auf Basis der DGE-Empfehlungen der Verbrauch von Milcherzeugnissen und Eiern deutlich gesenkt, der Fleischkonsum etwa halbiert werden. Legt man andere Maßstäbe zugrunde, wie beispielsweise die der Verfechter einer rein vegetarischen oder veganen Ernährung, empfiehlt sich eine weitere Reduktion tierischer Nahrungsmittel beziehungsweise ein kompletter Verzicht auf diese.

\section{Fazit}

Zusammenfassend ist die Erzeugung von Nahrungsmitteln tierischen Ursprungs zum einen ressourcenökonomisch ineffizient. Zum anderen ist der aktuelle, durchschnittliche Konsum von Fleisch, Milch und Eiern in Deutschland aus rein gesundheitlicher Sicht deutlich $\mathrm{zu}$ hoch. Sowohl aus individueller als auch aus gesellschaftlicher Perspektive kann vor diesem Hintergrund eine Reduktion des Verbrauchs von Nahrungsmitteln tierischen Ursprungs als sinnvoll angesehen werden. Die damit einhergehende, effizientere Nutzung der Anbauflächen würde nicht zuletzt dazu führen, die Ernährungssicherheit weltweit nachhaltig zu erhöhen.

\section{Anmerkung}

Herrn Alex Tschan sei an dieser Stelle herzlich für wertvolle Recherchetätigkeiten gedankt.

\section{Literatur}

BMEL, Bundesministerium für Ernährung, Landwirtschaft und Verbraucherschutz (2013): Statistisches Jahrbuch über Ernährung, Landwirtschaft und Forsten der Bundesrepublik Deutschland. Münster-Hiltrup, Landwirtschaftsverlag.

DGE, Deutsche Gesellschaft für Ernährung (2014): DGE-Ernährungskreis. Im Internet unter: http://www.dge.de/ernaehrungspraxis/ vollwertige-ernaehrung/ernaehrungskreis

Eshel G. et al. (2014): Land, irrigation water, greenhouse gas, and reactive nitrogen burdens of meat, eggs, and dairy production in the United States. In: Proceedings of the National Academy of Sciences of the United States of America 111/33, S. 11996-12001.

Esselstyn C. B. et al. (2014): A way to reverse CAD [Coronal Artery Disease]? In: The Journal of Family Practice 63/7, S. 356-364.

FNR, Fachagentur für Nachwachsende Rohstoffe (2014): Tierfutter überwiegt bei der Verwertung der EU-Getreideernte. Im Internet unter: http://mediathek.fnr.de/tierfutter-uberwiegtbei-der-verwertung-der-eu-getreideernte.html

LEAD, Livestock, Environment and Development Initiative (2006): Livestock's Long Shadow Environmental Issues and Options. Im Internet unter: ftp://ftp.fao.org/docrep/fao/010/ a0701e/a0701e.pdf

Meier, T. et al. (2014): Balancing virtual land imports by a shift in the diet. Using a land balance approach to assess the sustainability of food consumption. Germany as an example. In: Appetite 74. S. 20-34.

UN, Vereinte Nationen (2012): SecretaryGeneral's Initial Input to the Open Working Group on Sustainable Development Goals. Im Internet unter: http:// sustainabledevelopment.un.org/content/ documents/1494sgreportsdgs.pdf

WFP, UN World Food Program (2014): World Food Programme: Hunger weltweit - Zahlen und Fakten. Im Internet unter: http://de.wfp.org/hunger/hunger-statistik

\section{AUTOR + KONTAKT}

Dr. Tobias Gaugler ist wissenschaftlicher Assistent im Forschungsbereich Nachhaltiges Management von (Agrar-) Rohstoffen der Universität Augsburg.

Institut für Materials Resource Management (MRM), Universität Augsburg, Alter Postweg 101, 86159 Augsburg. Tel.: +49 821 598-3050,

E-Mail: tobias.gaugler@mrm.uni-augsburg.de

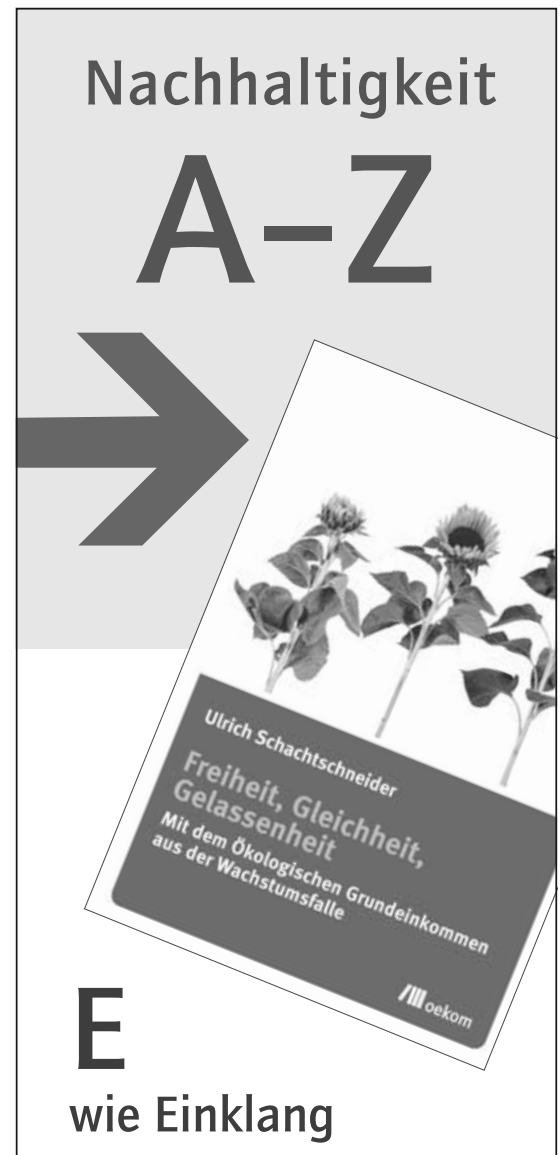

Soziale Sicherheit war bisher immer abhängig vom Wachstum. Doch die Praxis "Unten geben, ohne oben zu nehmen" kommt angesichts der Übernutzung natürlicher Ressourcen an ihr Ende. Und was nun? Ein Ökologisches Grundeinkommen ermöglicht nicht nur ein Leben im Einklang von Mensch und Natur. Über Ökosteuern finanziert, kommt es auch der sozialen Sicherheit zugute - ohne dabei bestimmte Lebensstile vorzuschreiben.

\section{U. Schachtschneider}

Freiheit, Gleichheit, Gelassenheit Mit dem Ökologischen Grundeinkommen aus der Wachstumsfalle

152 Seiten, broschiert, 16,95 Euro, ISBN 978-3-86581-693-1

Erhältlich bei

www.oekom.de

oekom@verlegerdienst.de

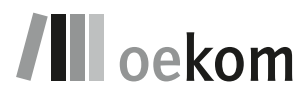

Die guten Seiten der Zukunft 